\title{
Lived Experience of Caregivers of Family-Centered Care in the Neonatal Intensive Care Unit: "Evocation of Being at Home"
}

\author{
Zahra Hadian Shirazi, ${ }^{1}$ Farkhondeh Sharif,, ${ }^{2}$ Mahnaz Rakhshan, ${ }^{2}$ Narjes Pishva, ${ }^{3}$ and Faezeh \\ Jahanpour ${ }^{4}$ \\ ${ }^{1}$ Department of Nursing, School of Nursing and Midwifery, Shiraz University of Medical Sciences, Shiraz, IR Iran \\ ${ }^{2}$ Community Based Psychiatric Care Research Center, Shiraz University of Medical Sciences, Shiraz, IR Iran \\ ${ }^{3}$ Neonatology Research Center, Shiraz University of Medical Sciences, Shiraz, IR Iran \\ ${ }^{4}$ School of Nursing and Midwifery, Bushehr University of Medical Sciences, Bushehr, IR Iran \\ "Corresponding author: Farkhondeh Sharif, Community Based Psychiatric Research Center, Shiraz University of Medical Sciences, Shiraz, IR Iran. Tel: +98-713647425051, Fax: \\ +98-7136474252, E-mail: farkhondeh_sharif@yahoo.com
}

Received 2015 August 31; Revised 2016 March 08; Accepted 2016 March 17.

\begin{abstract}
Background: In recent decades, family-centered care (FCC) has come to be known, accepted, and reported as the best care strategy for admitted children and their families. However, in spite of the increasing application of this approach, the experiences of the caregivers have not yet been studied.

Objectives: The present study aimed at the description and interpretation of the FCC experience in two neonatal intensive care units (NICU) at Shiraz University of Medical Sciences.

Methods: This study was conducted through the hermeneutic phenomenological approach. Semi-structured interviews were conducted with 17 professional and familial caregivers, and their interactions were observed in three work shifts. The interviews were audiotaped and transcribed verbatim. After observations, field notes were also written. Finally, the data were analyzed through van Manen's methodology.

Results: One of the essential themes that emerged in this study was the "evocation of being at home" among familial and even professional caregivers. This theme had three subthemes: i.e., "meta-family interaction," "comprehensive support," and "reconstruction of a normal family." Accordingly, FCC eliminated borders between professional and non-professional caregivers and built close relationships among them in the NICU. It also provided for the needs of neonates, their families, and even professional caregivers through perceived and received support.

Conclusions: Parents of the neonates admitted to the NICU experience hard moments. They not only play the role of primary caregivers, but they also receive the care. Focusing on the different meanings of this care from the caregivers' points of view and having managers provide certain requirements can guarantee the establishment of comprehensive care for clients and proper support for the staff in this unit.
\end{abstract}

Keywords: Family-Centered Care, Neonatal Intensive Care Unit, Hermeneutic Phenomenology

\section{Background}

The birth of a neonate is a magnificent and unique moment accompanied by celebrations and a lot of expectations for parents and families. Although dreams and ideals are made when a baby is born, the birth of a neonate that needs special care replaces deeply positive emotions with challenges and frustration for the family (1). The family is the constant in an infant's life, and his/her illness or discomfort affects all family members. The experiences in the neonatal intensive care unit (NICU) and its challenges may have a tremendous impact on a family's health (especially the mother) and its function in the long-run. Since nursing is a profession with a holistic approach to providing care, nurses have to interact with patients' family members and sometimes even take care of them. Therefore, considering families' needs and meeting them in the NICU are both essential (2-6).

In recent years, determined efforts have been made in order to reinforce care approaches or models for fulfilling neonates' and parents' needs. In this regard, familycentered care (FCC) has come to be known, accepted, and reported as the best strategy to take care of admitted children and their families (5). The American Academy of Pediatrics (AAP) defines FCC as an approach toward healthcare which encompasses policies, programs, facilities, and daily interactions among patients, families, physicians, 
nurses, and other members of the healthcare team (710). In the literature, various definitions have been provided for FCC, which affects its application in the clinical field. Despite the current challenges in settling on the best definition, conceptualization, and implementation of FCC, it is considered to be the standard for proper care in the NICU, or even the best-qualified approach in taking care of neonates at present. Different studies have indicated that the application of FCC had a lot of benefits for neonates, families, and health systems (10-14). The consequences of FCC in NICU include supporting families' mental health (e.g., decreasing the signs of stress, anxiety, and depression and developing self-esteem in those taking care of neonates at the hospital or in the home), facilitating attachment between parents and neonates, decreasing neonates' lengths of hospital stays and requirements for mechanical ventilation, decreasing some complications such as bronchopulmonary dysplasia, promoting neonates' overall health, and increasing parents' satisfaction with care and the personnel's satisfaction due to increases in their chances of being employed to work in the $\operatorname{NICU}(6,15-18)$.

In spite of the benefits of FCC in the NICU, its advantages are still questionable in non-western cultures and developing countries where families seldom get involved in the field of care $(1,19)$. Yet, professional members of healthcare teams in western countries have become acquainted with FCC through publications from developed countries, and may find it ideal for taking care of families in their own context. However, they are faced with some challenges, including the lack of sufficient resources and personnel, and the dominance of the established medical approach to the care process, thus prohibiting parents from making certain decisions $(14,19)$.

In Iran, families have become gradually more involved in the clinical context of the NICU through programs such as Kangaroo Mother Care. In addition, the elements of FCC, including respect, information sharing, participation, and collaboration, has been taken into more consideration in NICUs. Nevertheless, since one of the present study's researchers had the experience of working as both a nurse and head nurse in an NICU, she came to the conclusion that no real understanding of FCC existed in this particular unit. A review of the literature also revealed that no studies on determining and understanding the experience of FCC in neonatal intensive care in Iran had been published.

\section{Objectives}

Consequently, the present study aims to recognize professional and lay caregivers' experiences of FCC in an NICU.

\section{Methods}

The purpose of this study was to describe and interpret the experiences of caregivers involved in FCC in an NICU. This qualitative research was conducted according to the principles of hermeneutic phenomenology. Qualitative study is a psychological and systematic approach, which aims to describe the experience of life in an effort to make it meaningful (20). Phenomenology, as a qualitative research method, is meant to uncover humans' lived experiences of different phenomena, and to discover and explain pivotal phenomena of a particular discipline (21). Hermeneutic phenomenology attempts to describe the meanings hidden within our actions and experiences (22). The experience of FCC is a heavily mental one that needs to be extensively interpreted. Thus, here it is studied via hermeneutic phenomenology through van Manen's approach. This approach combines Husserlian descriptive phenomenology with a focus on universal study before pre-reflection. It contains an interpretive step as well (23). Van Manen has introduced several themes of the methodology which provide practical approaches in the implementation of hermeneutic phenomenology. He states that although he may present a special order, the practical approach of the research may be done simultaneously or intermittently in different directions (22). The themes of van Manen's methodology are described with respect to the researchers' activities as follows:

(a) Turning to the nature of lived experience: The researcher should recognize his/her phenomenon of interest and pose a phenomenological question. The important point is that he/she keeps the main question constantly in his/her mind during the study process. In the current study, the researchers selected their preferred phenomenon, i.e., FCC in NICU, and asked themselves, "What is the meaning of FCC in NICU?"

(b) Investigating the experience as we live it: In this stage, the researcher seeks to examine the lived experience of the phenomenon in any possible place, and commences from his/her own experience (22). In the current study, the primary researcher first wrote about her own personal experience by describing a special event. Then, she went to the field.

(c) Reflecting on the essential themes characterizing the phenomenon: In this stage, the researcher reflects on the lived experience, so that he/she can perceive the essential meaning of the experience. The primary researcher applied three approaches to thematic analysis, namely, holistic, selective, and detailed reading for isolating the themes (22).

Three additional themes are described in one of the sections below. 
Field and participants of the study: This study was conducted in two NICUs affiliated with Shiraz University of Medical Sciences. After obtaining formal consent, the primary researcher interviewed 17 professional and familial caregivers, including three neonatologists, six nurses, one social worker, six mothers, and one grandmother, selected through purposive sampling. All of these individuals had relevant lived experiences and took part in semi-structured interviews with the researcher. In addition, the researcher directly observed interpersonal interactions during three morning and evening shifts. The inclusion criteria for familial caregivers were: (1) neonate's hospitalization for at least one week, (2) stability of the neonate's and participant's physical conditions, (3) having a certain level of expressive ability, and (4) being willing to participate in the study. In addition, the inclusion criteria for professional caregivers were: (1) having at least six months of clinical working experience, (2) having a certain level of expressive ability, and (3) being willing to take part in the study.

Data collection: All of the interviews were performed in a calm place in the NICU where the participants felt comfortable. The interviews were started with the main question of the study. The participants were asked about the meaning of FCC. Afterwards, the researcher used explorative questions and statements, such as "Can you explain more?", "Explain any memories about that," and, "When you say [x], what do you mean?" The interviews lasted for 31 to 75 minutes. For each participant, two interviews were held within two sessions. The researcher listened to the recordings of the interviews and wrote them out verbatim. Data gathering and analysis were done simultaneously. According to van Manen, the best way for entering a person's lived world is through participation in that world, which requires close observation as both an observer and a participant at the same time (22). In the current study, observations were done within three shifts, and the researcher provided information and assistance to the caregivers if necessary. Finally, after the meaning of FCC was clear to all of the involved researchers, they searched for texts and articles to find lived experiences in any other possible place. Eventually, they used a narrative of a mother who had a premature neonate in NICU who had written about her experience with FCC (24).

Thematic analysis: Adopting the holistic approach, the primary researcher considered the text as a whole after reading it and then writes down its essential meaning. In the selective approach, she selected the statements, phrases, or groups of statements that seemed to be essential for describing the experiences after reading the texts several times. In the detailed approach, the researcher asked what parts of the described phenomenon or expe- rience could have been clarified. She asked this question while confirming every statement or a group of statements. She made an effort to recognize thematic expressions and phrases that provided the phenomenological meaning of the experiences. In this stage, van Manen recommends formal and informal sorts of participatory analysis (22). It should be noted that from the first stage of the study, the researcher used the opinions of her professor, supervisors, and external supervisor, and presented the research's results in a formal seminar. The essential and incidental themes were determined by use of free imaginative variation, and one essential theme has been presented here.

Additional themes from van Manen's methodology are as follows:

(d) Describing the phenomenon through the art of writing and rewriting: The structure and meaning of the lived experience can be discovered through writing and rewriting. The findings and discussions of the current study will determine whether this has been achieved in this research.

(e) Maintaining a strong and oriented relationship with the phenomenon; van Manen believes that a phenomenological text must be deep, rich, strong, and oriented (22). Reviewing the findings of the study will show the extent of the strong and oriented relationship between the research and the phenomenon.

(f) Balancing the research context by considering the parts and the whole: According to van Manen, the researcher should monitor the general design of the study continuously in addition to considering the importance of details in the general context. He introduces different ways of organizing a phenomenological text (22). The thematic method was applied for describing and interpreting the research in the current study.

Ethical consideration: This study was approved by the Ethics Committee of Shiraz University of Medical Sciences. The primary researcher also referred to the standards of the field and obtained the necessary permissions. Additionally, she informed the participants about the study process and their rights both orally and in written form, and then obtained their written informed consent. The researcher made sure that they were aware that they could withdraw from the study whenever they wanted without interfering with their neonates' treatment processes. Confidentiality was assured through the anonymity of the participants.

Trustworthiness of the study: Lincoln and Guba's criteria, including credibility, dependability, confirmability, and transferability, are used to determine trustworthiness in qualitative studies (25). In the current study, triangulation of data collection methods was applied, and the re- 
searcher had prolonged engagement with the data. Moreover, descriptive anecdotes of the lived experiences were shown to and confirmed by the participants (26). In addition, participatory analysis was done officially by the professor, supervisors, and the external supervisor. Transferability of the study must be evaluated by the readers. Ultimately, the researcher was responsible for providing thick description (25).

\section{Results}

In the present study, the experiences of professional and familial caregivers at the NICU regarding the phenomenon of FCC phenomenon were studied, and the essential themes were extracted. One of these essential themes was "evocation of being at home" with three subthemes, namely, "meta-family interaction," "comprehensive support," and "reconstruction of a normal family."

The hospitalization of a neonate in an NICU is stressful for a family and may disturb its functions. In this respect, FCC prepares parents for playing their parental roles in such circumstances. In contrast to what was believed in the past, the presence of a caregiver is respected and accepted by families, and fulfills their needs through communication that may be similar to their familial style (17). In this study, familial caregivers experienced their presence in the NICU as similar to their presence at home and having communication with their own family members. Moreover, they perceived and received comprehensive support through this communication. Furthermore, professional caregivers perceived and received support in relation to the family and their colleagues.

The meta-family interaction subtheme included "emotional identification with somebody" and the "persistence of therapeutic communication." A nurse spoke about emotional communication, saying, "We really had no nerve to transfer her to another ward, you know we didn't have the heart to do it, we loved her so much." Furthermore, another nurse intended to keep in touch with the neonate after discharge. She said, "If, for example, it was arranged that we visited the baby more after discharge or it was arranged that, for example, the mother would come and the personnel could see the baby it would be great, and we would be less worried about the baby"

Some of the participants even went beyond this level of engagement and talked about the reciprocal feeling of being a member of the family. One nurse stated, "We feel a maternal role in ourselves. On many occasions, I say 'my darling,' 'my daughter,' or I see that a nurse says 'my honey,' 'I love you"' Also, one of the mothers mentioned, "Every one of these nurses was considered to be a main member of our family."
The parents kept in touch with the NICU nurses after discharge for solving their neonates' therapeutic problems, and even informed the nurses about their general conditions. At the same time, the personnel embraced this relationship enthusiastically. One of the nurses said, "When a baby that is in a ward like the NICU is discharged, his/her care is not finished. It is still going on. We followup to know how he/she is taken care of and we follow him/her."

Both professional and familial caregivers emphasized the experience of "comprehensive support" through FCC. They believed in this support and declared that they received it completely. The belief in the existence of this support among familial caregivers was expressed in the form of describing a shared feeling, causing them to feel calm. One of the mothers said, "Some nurses really took care [of my baby) very well. That was a weight off my mind. For example, when I was going home. I was feeling that if I wasn't there, she was taken care of very well." Another mother also mentioned, "I felt easy in my mind that he was here because I saw their care and I trusted them."

Even professional caregivers believed that they were supported by familial caregivers and their intra/interprofession colleagues. One nurse said, "Her baby is connected to a pulse oximeter. I put her baby in her arms with two other babies whose mothers are absent now, and I should really do their deeds. I feel ease in my mind knowing that he/she is in his/her mother's arms, and if something happens the mother lets me know. I do my other tasks." Another nurse also said, "We enjoy it a lot when our special physician relies on us and even expresses it."

The familial caregivers' experiences of receiving comprehensive support, including informational, spiritual, psychological, religious, and cultural support, were tangible. One mother talked about receiving informational support from the physician, saying, "When you ask any questions, he answers. Then, he, for example, explains everything to you clearly. When you're at your patient's bedside he describes that she has this problem, for instance, so I don't discharge her because of this problem."

One of the main needs of the parents is psychospiritual support, which can facilitate adaptation to the neonate's hospitalization and enhance the ability to handle difficult situations. In this regard, one mother maintained, "I was going to my son's bedside, and I was really crying, but the personnel told me not to cry there because he gets it, we do what is necessary for him, he'll be ok These words made me calm and relaxed."

With respect to financial support for families, a social worker stated, "We could take a 16 million tomans. The remaining amount is about 12 million tomans, which they can pay in installments." 
Professional personnel also receive psycho-spiritual and informational support from each other. One of the physicians said, "We try, like today, to hold classes every Thursday and give them (the nurses) new information so as to involve them in patients' affairs. I say that my own belief is that the awareness of babies' nurses must be more than that of their doctors."

"Reconstruction of a normal family" as the third study subtheme included "welcoming of presence, stay, and participation of familial caregivers in the NICU" and "preparing to be a parent." The participants talked about the enthusiastic acceptance of caregivers from families. One of the physicians said, "I personally say if a baby's father and mother don't come to the hospital for 2 - 3 days. I surely say that this baby is like Hutch the Honeybee (laughing), you know, because it's very important for me that they get involved in their baby's affairs and I follow-up on the situation." Additionally, a mother stated, “ When I sat outside for just about five minutes, they called me and told me my baby was crying, and if I would like, I could go and embrace him and calm him down. Whenever he was crying, they did me this favor."

After a neonate's hospitalization and his/her isolation from the family, one of the main problems for parents, especially mothers, is the disturbance of the parental role (27). FCC provides the groundwork for the parents to recognize their own distinctive and irreplaceable role as a parent. One of the mothers under study mentioned, "From that day, Mrs. [X] encouraged me to do her chores up to now, and I feel like a mother. I mean, when I didn't do her chores, I didn't feel that I was her mother."

\section{Discussion}

The current study aimed at describing and interpreting the experiences of the caregivers working in NICUs about FCC. Family, as the constant in an infant's life, is known as his/her main supportive source and primary caregiver $(28,29)$. Therefore, the experiences of both familial and professional caregivers were investigated in this study. One essential theme that emerged in this study was the "evocation of being at home" with three subthemes, namely, "reconstruction of a normal family," "meta-family interaction," and "comprehensive support." The results showed that meta-family interaction was established through FCC. Cultivating deep emotional relationships between the personnel and the neonates (and sometimes their mothers) established enjoyable experiences, so that there was eagerness to preserve this reciprocal relationship even after the neonates' discharge. Fegran et al. (2009) (29) stated in their study that the parents described their relationship with nurses as a sort of contact they might have with their close friends. However, their study indicated that nurses, in such a situation, tried to limit their relationships in order not to act out of their professional roles. They also believed that in spite of the usefulness of closer relationships, the emotional load of these relations could be problematic and create obstacles against parents' independence and adaptation to new conditions. These results were in contrast to those of our investigation. This may be due to the difference between the cultural backgrounds of the two studies. Fegran et al. (2008) discussed the recognition of FCC experience in different backgrounds (30).

In this study, professional caregivers were eager to maintain their relationships with the neonates' families. They were concerned about the neonates and were eagerly interested in receiving the parents' contact in order to know more about their problems and provide them with possible solutions. In addition, the mothers confessed that keeping in touch with professional caregivers encouraged them to take better care of their neonates even after achieving independence in caregiving. They believed that this relationship resulted in the development of their parental capabilities. This might be attributed to the lack of homecare in Iran's health system. In fact, the establishment of friendly and intimate relationships between familial and professional caregivers is a part of the essence of FCC, which has been proven to be successful in the current study and other research. Nevertheless, approaches towards the acceptance of such relationships differ in various contexts, which are specifically related to different caring policies and facilities.

The findings of the present study indicated that FCC was experienced as a unique phenomenon for supporting the family, especially the mother, and even professional caregivers. Both neonates' and families' needs are taken into consideration in FCC. In other words, not only is the family regarded as one of the neonate's care providers, but it is also considered to be the receiver of the care as well (15). In the study by Molina et al. (2009), the parents expressed that they were taken care of in addition to giving care to their neonates. They believed that the care in the NICU included performing medical techniques and procedures as well as expressing certain feelings and attitudes, such as care, attention, and benevolence (4). In the research by Aliabadi et al. (2014) (31), the supportive-emotional needs of the Iranian parents with hospitalized premature neonates included the need for interaction with the neonate, the treatment team's empathy, exchange of support with the couple, and the seeking of help from others.

Based on the present results, the supports provided for families according to the parents' needs consisted of informational, psycho-spiritual, physical, religious, network- 
ing, and financial support. The professional staff did their best to provide the necessary support for families, especially mothers. Nevertheless, there were some obstacles, including heavy workloads and lack of time, which might hinder the provision of this kind of care. Trajkovski et al. (2012) (5) stated that although the nurses' main concern was each neonate's health, taking care of families was also important. Moreover, nurses required continuous support, guidance, and training from their organizations to foster the provision of efficient FCC. One of the findings of the present study was the belief in being supported by the parents and nursing staff. Perceived support on behalf of the staff promotes the level of occupational performance and decreases occupational stress among nurses (32). Furthermore, in the research performed by Aliabadi et al. (2014) (31), the parents under study confessed that their stress and negative feelings, even when they were present at their babies' bedside, decreased due to the quality of care provided by the staff. This indicated their belief in the existence of support.

The results of the present study have proven the role of FCC in the reestablishment of a family's stability. Wigert et al. (33) (2014) stated that the parental process begins in the NICU as an involuntary journey with the final goal of a family's efficient performance. Encouraging the parents to take care of their neonates and facilitating trust-centered communication enabled them to establish their parental identities. Molina et al. (2009) (4) declared that a family's dynamics can change due to neonate's hospitalization. Fear, anxiety, and loneliness resulting from a neonate's hospitalization can disturb the balance of parental roles, putting the family structure in danger. However, using FCC elements protected the family structure and enabled families to reconstruct their capabilities and keep their own integrity. Other studies have also shown that the presence of parents and their involvement in taking care of the neonates led to better acceptance of the parental role, recognizing its impact on his/her life, and having the feeling of a united family (27, 28, 34-36).

Considering the fundamental role of family in the neonate's life and the capacity of this approach for providing the best sort of care in the NICU, it is suggested that the philosophy and policies of an NICU be structured around FCC. In addition, nursing students, and especially staff, should be educated and retained, and their performance has to be continually assessed. Moreover, the obstacles against implementing FCC must be recognized in the context through a participatory approach to research. Then, such obstacles have to be eliminated through the cooperation of managers, staff, and families. It is also recommended that a homecare program be implemented as part of the post-discharge plans.
According to the current study's findings, the knowledge and practice applied in the NICU can be promoted in clinical, educational, and research fields. However, since the experiences of a limited number of participants were used in this study, the results might not be generalizable. Nonetheless, the goal of qualitative studies is not only the generalization of the results, and this is the instinctive feature of this kind of research (25).

\subsection{Conclusion}

According to the results of the present study, "evocation of being at home" was an essential part of the caregivers' experience in the NICU involving FCC. Indeed, FCC in this ward was a unique phenomenon which was able to protect and reconstruct the foundation of the family in the difficult situation caused by a neonate's hospitalization and his/her involuntary isolation from the family. In this model of care, success could be achieved through comprehensive support and the establishment of meta-family interactions. This sort of care could also provide comprehensive support for professional caregivers as well.

\section{Acknowledgments}

The authors would like to thank Ms. A. Keivanshekouh at the research improvement center of Shiraz University of Medical Sciences for improving the use of English in the manuscript. They are also grateful for Hazrat-e-Zeynab (P.B.U.H.) hospital authorities and the center for development of clinical research, Namazee hospital, Shiraz, Iran for their cooperation. Thanks also go out to professional caregivers and families for their kind cooperation in the study. The authors would also like to extend their appreciation to Professor Max van Manen and Dr. Michel van Manen who answered their questions humbly. Finally, they would like to thank Dr. Kamelia Torabizadeh, Professor Hamid Peiravi, and Dr. Zarintaj Varedi for their cooperation.

\section{Footnotes}

Authors' Contribution:Zahra Hadian-Shirazi: developed the original idea and protocol; collected, abstracted and analyzed data; wrote the manuscript; Farkhondeh Sharif: developed the original idea and protocol; supervised the data collection and analysis; supervised manuscript writing; Mahnaz Rakhshan: developed the original idea and protocol; supervised the data collection and analysis; supervised manuscript writing; Narjes Pishva: developed the original idea and protocol; supervised manuscript writing; Faezeh Jahanpour: developed the original idea and protocol; supervised the data collection and analysis. 
Funding/Support: This study was financially supported by the research vice-chancellor of Shiraz University of Medical Sciences (Grant No. 92-6901).

\section{References}

1. Martins LA, Silva DS, Aguiar ACSA, Morais AC. Insertion of the Family in the Neonatal Intensive Care Unit: A Systematic Review. J Nurs UFPE on line. 2012;6(4).

2. Frost M, Green A, Gance-Cleveland B, Kersten R, Irby C. Improving family-centered care through research. J Pediatr Nurs. 2010;25(2):1447. doi: 10.1016/j.pedn.2009.09.001. [PubMed: 20185066].

3. McKiernan M, McCarthy G. Family members' lived experience in the intensive care unit: a phemenological study. Intensive Crit Care Nurs. 2010;26(5):254-61. doi: 10.1016/j.iccn.2010.06.004. [PubMed: 20674362].

4. Molina RC, Fonseca EL, Waidman MA, Marcon SS. [The family's perception of its presence at the pediatric and neonatal intensive care unit]. Rev Esc Enferm USP. 2009;43(3):630-8. [PubMed: 19842596].

5. Trajkovski S, Schmied V, Vickers M, Jackson D. Neonatal nurses' perspectives of family-centred care: a qualitative study. J Clin Nurs. 2012;21(17-18):2477-87. doi: 10.1111/j.1365-2702.2012.04138.x. [PubMed: 22889445].

6. Manning AN. The NICU experience: how does it affect the parents' relationship?. J Perinat Neonatal Nurs. 2012;26(4):353-7. doi: 10.1097/JPN.ob013e3182710002. [PubMed: 23111724].

7. Hockenberry MJ, Wilson D. Wong's Nursing Care of Infants and Children. 9 ed. Mosby: Elsevier; 2011.

8. Gibbins S. Pain management: What is the Role of the Parent. Pain Column. 2008;8(3):116-7.

9. Griffin T, Abraham M. Transition to home from the newborn intensive care unit: applying the principles of family-centered care to the discharge process. J Perinat Neonatal Nurs. 2006;20(3):243-9. [PubMed: 16915057].

10. Conway J, Celenza J, Abraham M. Advancing patient- and familycentered newborn intensive care. Burlington: Vermont Oxford Network; 2007.

11. Nichols A. Sustainable family centred care in the neonatal unit. $J$ Neonat Nurs. 2013;19(5):266-70.

12. Fegran L. Parents and Nurses in a Neonatal Intensive Care Unit- The Development of a Mutual benefical Partnership In the Care of the Infant. Oslo: The University Foundation for Student Life (SiO); 2009.

13. Cooper LG, Gooding JS, Gallagher J, Sternesky L, Ledsky R, Berns SD. Impact of a family-centered care initiative on NICU care, staff and families. J Perinatol. 2007;27 Suppl 2:32-7. doi: 10.1038/sj.jp.7211840. [PubMed: 18034178].

14. Staniszewska S, Brett J, Redshaw M, Hamilton K, Newburn M, Jones $\mathrm{N}$, et al. The POPPY study: developing a model of family-centred care for neonatal units. Worldviews Evid Based Nurs. 2012;9(4):243-55. doi: 10.1111/j.1741-6787.2012.00253.x. [PubMed: 22698274].

15. Ramezani T, Hadian Shirazi Z, Sabet Sarvestani R, Moattari M. Familycentered care in neonatal intensive care unit: a concept analysis. Int J Community Based Nurs Midwifery. 2014;2(4):268-78. [PubMed: 25349870].

16. Ortenstrand A, Westrup B, Brostrom EB, Sarman I, Akerstrom S, Brune T, et al. The Stockholm Neonatal Family Centered Care Study: effects on length of stay and infant morbidity. Pediatrics. 2010;125(2):278-85. doi: 10.1542/peds.2009-1511. [PubMed: 20100748].

17. Gooding JS, Cooper LG, Blaine AI, Franck LS, Howse JL, Berns SD. Family support and family-centered care in the neonatal intensive care unit: origins, advances, impact. Semin Perinatol. 2011;35(1):20-8. doi: 10.1053/j.semperi.2010.10.004. [PubMed: 21255703].
18. Ladak LA, Premji SS, Amanullah MM, Haque A, Ajani K, Siddiqui FJ. Family-centered rounds in Pakistani pediatric intensive care settings: non-randomized pre- and post-study design. Int J Nurs Stud. 2013;50(6):717-26. doi: 10.1016/j.ijnurstu.2012.05.009. [PubMed: 22704527].

19. Shields L. Questioning family-centred care. J Clin Nurs. 2010;19(1718):2629-38. doi: 10.1111/j.1365-2702.2010.03214.x. [PubMed: 20920083].

20. Oskouie F, Peyrovi H. Pazhouheshe keyfi dar parastari [In persian]. 1 ed. Iran,Tehran: Hamrah Publishing; 2005.

21. Streubert HJ, Carpenter DR. Qualitative Research in Nursing: Advancing the Humanistic Imperative. 5 ed. Philadelphia: Lippincott Williams\& Wilkins; 2011.

22. Van Manen M. Researching Lived Experience: Human Science for an Action Sensitive Pedagogy. 2 ed. Canada: The University of Alberta; 2001.

23. Dowling M. From Husserl to van Manen. A review of different phenomenological approaches. Int J Nurs Stud. 2007;44(1):131-42. doi: 10.1016/j.ijnurstu.2005.11.026. [PubMed:16412442].

24. Van Dell T. Patient- and family-centered care in the neonatal intensive care unit in our own words: one family's story. AACN Adv Crit Care. 2011;22(4):298-300. doi: 10.1097/NCI.0b013e31822ca54b. [PubMed: 22064577].

25. Polit DF, Beck CT. Nursing Research: Generating and Assessing Evidence for Nursing Practice. 9 ed. Philadelphia: Lippincott Williams \& Wilkins; 2012

26. van Manen M. Phenomenology of practice: Meaning-giving methods in phenomenological research and writing. Left Coast Press; 2014

27. Kearvell H, Grant J. Getting connected: How nurses can support mother/infant attachment in the neonatal intensive care unit. Aust $J$ Adv Nurs. 2010;27(3):75-82.

28. Beck SA, Weis J, Greisen G. Room for family-centered care - a qualitative evauation of a neonatal intensive care unit remodeling project. $J$ Neonat Nurs. 2009;15(3):88-99.

29. Fegran L, Helseth S. The parent-nurse relationship in the neonatal intensive care unit context-closeness and emotional involvement. Scand J Caring Sci. 2009;23(4):667-73. doi: 10.1111/j.14716712.2008.00659.x. [PubMed: 19000088].

30. Fegran L, Fagermoen MS, Helseth S. Development of parent-nurse relationships in neonatal intensive care units-from closeness to detachment. $J$ Adv Nurs. 2008;64(4):363-71. doi: 10.1111/j.13652648.2008.04777.x. [PubMed: 18783472].

31. Aliabadi F, Kamali M, Borimnejad L, Rassafiani M, Rasti M, Shafaroodi $\mathrm{N}$, et al. Supporting-emotional needs of Iranian parents with premature infants admitted to Neonatal Intensive Care Units. Med J Islam Repub Iran. 2014;28:53. [PubMed: 25405119].

32. AbuAlRub RF. Job stress, job performance, and social support among hospital nurses. J Nurs Scholarsh. 2004;36(1):73-8. [PubMed: 15098422].

33. Wigert H, Dellenmark Blom M, Bry K. Parents' experiences of communication with neonatal intensive-care unit staff: an interview study. BMC Pediatr. 2014;14:304. doi: 10.1186/s12887-014-0304-5. [PubMed: 25492549].

34. Erlandsson K, Fagerberg I. Mothers' lived experiences of co-care and part-care after birth, and their strong desire to be close to their baby. Midwifery. 2005;21(2):131-8. doi: 10.1016/j.midw.2004.09.008. [PubMed: 15878428].

35. Malakouti J, Jebraeili M, Valizadeh S, Babapour J. Mothers' experience of having a preterm infant in the Neonatal Intensive Care Unit, a phenomenological study. J Crit Care Nurs. 2013;5(4):172-81.

36. Lundqvist P, Westas LH, Hallstrom I. From distance toward proximity: fathers lived experience of caring for their preterm infants. JPediatr Nurs. 2007;22(6):490-7. doi:10.1016/j.pedn.2007.04.008. [PubMed: 18036470]. 